\title{
Potential Docking Affinity of three Approved Drugs against SARS-CoV-2 for COVID-19 treatment.
}

Venkata Sambasiva Rao Rachakulla ${ }^{1}$, Hemanjali Devi Rachakulla²

${ }^{1}$ Department of Math, Greene County High School, Greensboro, GA, 30642 USA.

${ }^{2}$ Department of Science, Jonesboro High School, Jonesboro, GA, 30236, USA.

${ }^{1}$ Author for correspondence email: rachakullav@gmail.com

${ }^{2}$ Author email: hemanjali27@gmail.com

\section{Abstract}

Objectives: The availability of a safe and effective drug for COVID-19 is well-recognized as an additional tool to contribute to the control of the pandemic. At the same time, the challenges and efforts needed to rapidly develop, evaluate, and produce this at scale are enormous. It is vital that we evaluate as many vaccines as possible as we cannot predict how many will turn out to be viable.

Methods: In this study, we have measured the virtual interaction of crystal data structures of protein downloaded from protein data bank (PDB ID 7BRP) with corticosteroid drug candidates approved by FDA for other medical purposes which have less side effects. The results are analyzed in contrast some drugs candidates currently using for the treatment of COVID-19.

Results: The binding energies in kilocalories/mole obtained from the docking of 7BRP protease with ligands under investigation Betamethasone Phosphate (-6.9), Fluticasone (-6.1) and Dexamethasone (-5.9) and also with currently using drug candidates Remdesivir(-6.5), Lopinavir (-6.0), Baceprivir(-5.7), Rabavirin(-6), Ritinovir(-5.3), Hydroxyquinoline(-5.0), Chloroquine (-4.7), Oseltamivir(-4.6), Favipiravir(-3.9).

Discussion: The docking results suggest a higher binding affinity of the drug molecules under investigation against SARS-CoV-2 in contrast with other drug candidates currently being used for 
the treatment of COVID-19. We have analyzed bond interactions of protein-ligand from images in 10 modes of investigated drugs in contrast with Remdesivir and also discussed the advantages of inhalation methods of drug fluticasone.

Conclusion: From this study, it can be suggested that these drugs are promising candidates for antiviral treatment with high potential to fight against SARS-CoV-2 strain keeping in view various ways of administration of drugs currently practicing.

Keywords: FDA approved drugs, Docking, SARS-CoV-2 protease, treatment methods.

\section{Introduction}

As on today 23nd June 2020 there are 8974765 COVID-19 cases worldwide and reported 469159 deaths and 216 countries, areas and territories affected according to the World Health Organization (WHO). In silico approach, docking has become a promising tool for drug discovery and development. We study virtual interaction of ligand (drug) molecules inside the binding pocket of target protein (receptor) [1]. It offers study of all the factors that are being utilized for drug discovery such as identification of hit molecules, optimization of lead compound and virtual screening. [2-5]. Since it has been reported that the several existing drug showed potential against COVID-19 including oseltamivir,[6] lopinavir,[7] ritonavir,[7] remdesivir,[8] favipiravir,[9] ribavirin,[9] chloroquine and hydroxychloroquine.[10] Since most of the drugs are HIV protease inhibitors[11] we have performed their docking with COVID-19 main protease downloaded from protein data bank (PDB ID 7BRP). Some of the Potential Therapeutic Target Against SARS-CoV and SARS-CoV-2 Several similarities between SARS-CoV-2 and SARS-CoV have been reported. Since the sequencing of SARS-CoV-2 has been done,[12] it has given rise to various molecular modeling experiments to find the potential candidate against novel 
coronavirus SARS-CoV-2. The SARSCoV-2 originating from bat has been revealed by the phylogenetic analysis. Xu et al. $[12,13]$ have performed some molecular modeling experiments which revealed similarity in the 3-D structures of SARS-CoV-2 and SARSCoV in the RBD (receptor binding domain). This leads to designing of various approaches to find out the potential target for development of potential drug candidate against SARSCoV-2. Molecular Docking Study of Some Drug Candidates with Protease of SARS-CoV2. Due to unavailability of specific treatment for COVID-19 are several antiretroviral drugs reported to show effectiveness against COVID-19 including ritonavir,[14, 15] lopinavir,[15] alone or in combination with oseltamivir,[15] remdesivir, chloroquine and hydroxychloroquine.[16]. Out of these, ritonavir, remdesivir, chloroquine and hydroxychloroquine has shown efficacy at cellular level [14] which will be evaluated in future by various experimental studies. In drug discovery, molecular docking methodologies is a useful tool as is rapid screening of candidates from drug libraries. [17, 18]. In this research, we have performed docking study of some of the potential therapeutic drug candidates that are being used against COVID-19 worldwide by using computational techniques. Methods of all the docking experiments were performed by using Auto Dock Vina extended on $1^{\circ}$ A Samson 2020 (software for adaptive modeling and simulation of nano systems),[19] because (a) it offers more accuracy in predicting ligand-protein interaction compared to its previous Auto Dock 4.2 (b) it offers shorter running time because of its multiple core processors (c) it offer more accuracy for ligand processing more than 20 rotatable bonds. All dockings were performed as blind dockings (blind docking refers to the use of a grid box which is large enough to encompass any possible ligand-receptor complex). All the protein structures used in docking experiments 
were retrieved from the protein data bank. All the conformational ligand structures were created by using smiles codes on Samson software to have the most stable structure using energy minimization for the force field and conjugate gradient method. The ligand and protein molecules were converted to their proper readable file format pdb files using Discovery Studio Visualizer 2020. The docking was done using an exhaustiveness value of 8 with a measurement of 10 modes each docking. All other parameters of software were kept as default and all bonds contained in ligands could rotate freely, considering the receptor as rigid. The final visualization of the docked structure was performed using Discovery Studio Visualizer 2020. The results obtained from these experiments indicated the strong interactions of the potential drug candidates against COVID-19 main protease.

Comparison of Binding affinities: After successful docking of these drugs into the COVID-19 main protease in complex, the results show various modes of drug-protein interactions are generated with docking score (binding energy). The structures of ligand molecules used for docking are shown in the Fig.2. The binding mode with least binding energy is regarded as the best mode of binding as it is most stable for the ligand. The binding energies of drug candidates under investigation in contrast currently used drugs for treatment purposes of COIVD-19 are summarized as Table 1 and also shown in the graph in Fig.1. From the binding energies in different modes, betamethasone phosphate has shown more bonding affinity with protein when compared to remedesivir and lopinavir. Other two drugs Fluticasone and Dexamethasone have also shown lesser binding affinity when compared to remedesivir but comparatively closer to lopinavir and ribavirin and more binding affinity than other drugs under investigation. 


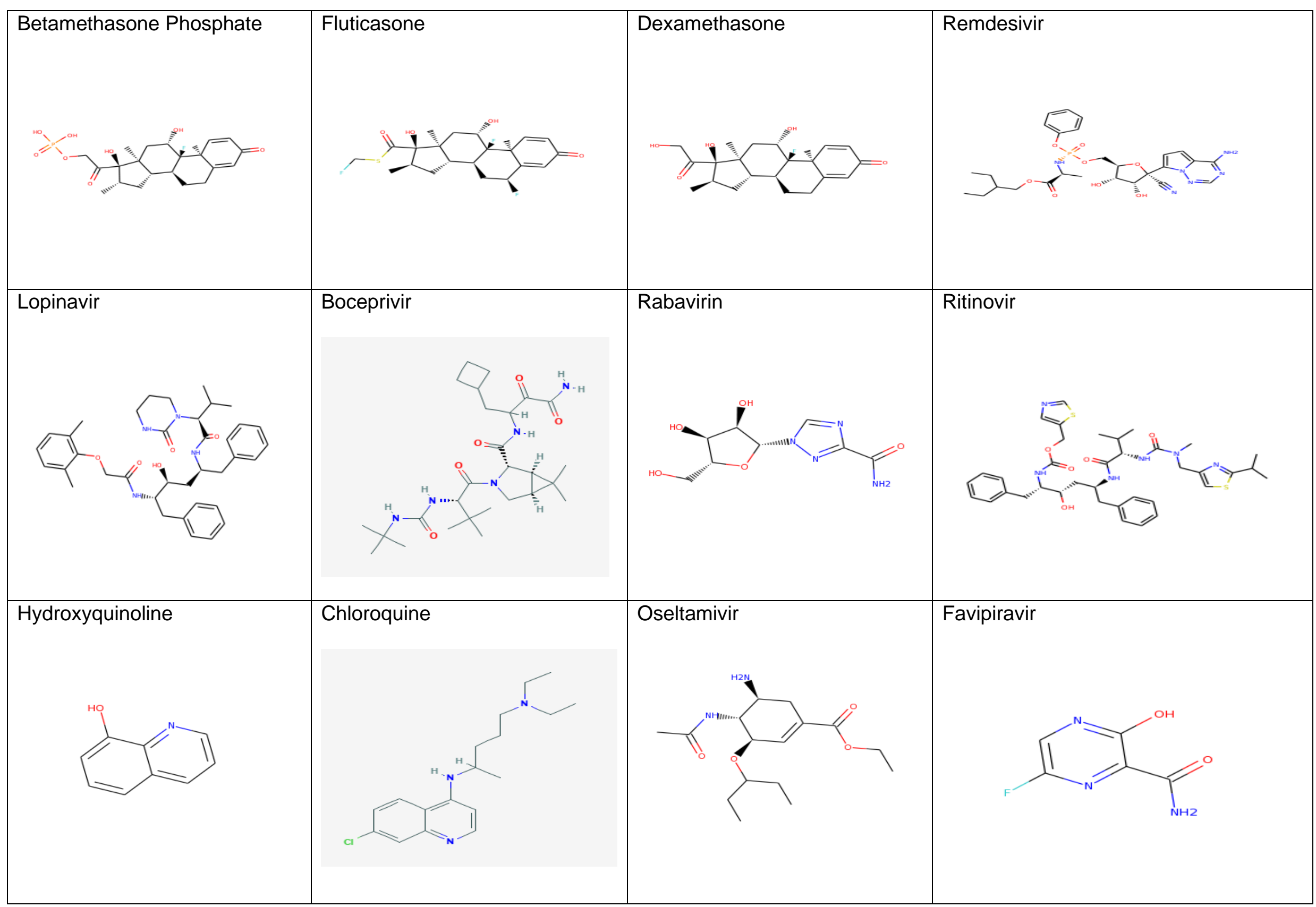

Figure 2: Chemical Structures of molecules docked with main protease of SARS-CoV-2 from protein data bank (PDB ID 7BRP) 


\begin{tabular}{|c|c|c|c|c|c|c|c|c|c|c|c|c|}
\hline Modes & $\begin{array}{l}\text { Betametha } \\
\text {-sone } \\
\text { phosphate }\end{array}$ & Remdesivir & Lopinavir & Fluticasone & $\begin{array}{l}\text { Dexameth } \\
\text { asone }\end{array}$ & Baceprivir & Ribavirin & Ritinovir & $\begin{array}{l}\text { Hydro } \\
\text { xyquin } \\
\text { oline }\end{array}$ & $\begin{array}{l}\text { Chloro } \\
\text { quine }\end{array}$ & Oseltamivir & Favipiravir \\
\hline 1 & -6.9 & -6.5 & -6 & -6.1 & -5.9 & -5.7 & -6 & -5.3 & -5 & -4.7 & -4.6 & -3.9 \\
\hline 2 & -6.8 & -6.4 & -5.9 & -5.9 & -5.8 & -5.5 & -6 & -5.3 & -5 & -4.6 & -4.5 & -3.9 \\
\hline 3 & -6.6 & -6.4 & -5.6 & -5.6 & -5.6 & -5.4 & -5.1 & -5.2 & -4.8 & -4.6 & -4.4 & -3.9 \\
\hline 4 & -6.5 & -6.3 & -5.5 & -5.6 & -5.6 & -5.3 & -5.1 & -5.1 & -4.8 & -4.5 & -4.4 & -3.8 \\
\hline 5 & -6 & -6.3 & -5.5 & -5.5 & -5.4 & -5.3 & -5 & -5 & -4.8 & -4.5 & -4.2 & -3.8 \\
\hline 6 & -5.9 & -5.8 & -5.5 & -5.4 & -5.2 & -5.2 & -4.9 & -5 & -4.7 & -4.4 & -4.2 & -3.8 \\
\hline 7 & -5.9 & -5.7 & -5.5 & -5.3 & -4.9 & -5.2 & -4.7 & -5 & -4.7 & -4.4 & -4.2 & -3.7 \\
\hline 8 & -5.8 & -5.7 & -5.4 & -5 & -4.9 & -5.2 & -4.7 & -5 & -4.6 & -4.2 & -4.1 & -3.7 \\
\hline 9 & -5.8 & -5.6 & -5.4 & -4.9 & -4.9 & -5 & -4.6 & -4.9 & -4.6 & -4.2 & -4.1 & -3.7 \\
\hline 10 & -5.7 & -5.6 & -5.3 & -4.8 & -4.7 & -5 & -4.5 & -4.9 & -4.6 & -4.1 & -4 & -3.7 \\
\hline
\end{tabular}

Table 1: Binding affinity of ligands in all 10 modes in kcalories. mole ${ }^{1}$ 


\section{Binding affinities in all 10 modes for each ligand}

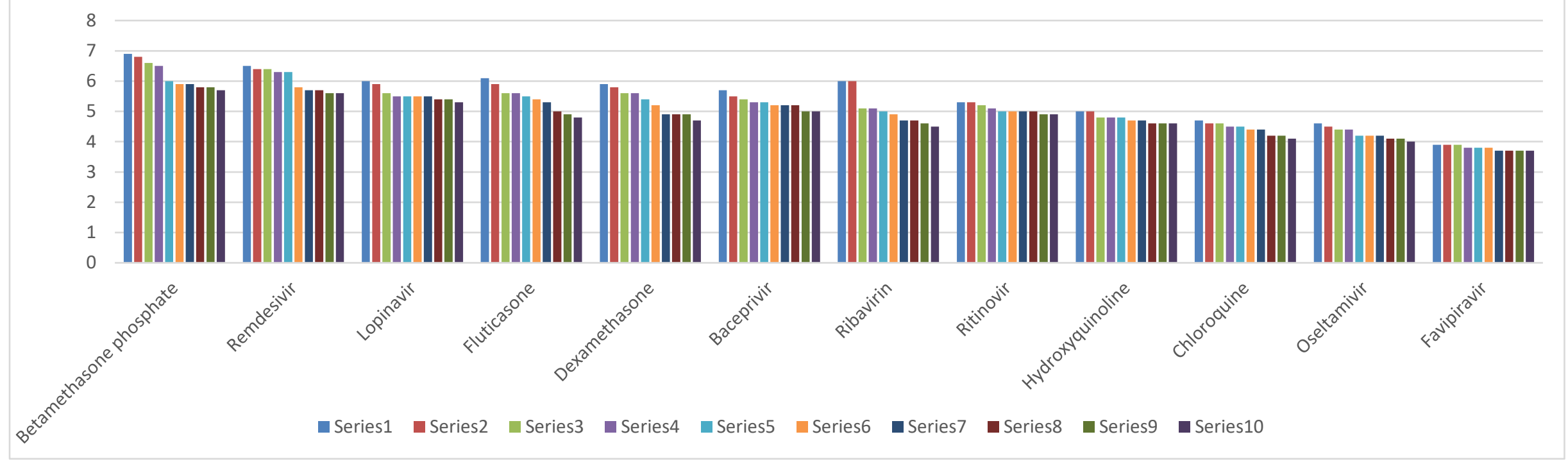

Figure 1: Binding affinity graph of all ligands in all 10 modes for each ligand. (Positive values are taken for graphical comparison purpose 
Comparison of protein-ligand binding interactions with graphs: The protein-ligand binding interactions are studied for selected comparable drug candidates like betamethasone phosphate, fluticasone, dexamethasone with remedesivir. It is also revealed the interaction of specific amino acids that take part in the drug-protein interactions. All the docked structures were visualized by Discovery Studio Visualizer 2020. The visualized 3D pictures of sitting of ligand in protein structure and 2D pictures of bonding interactions are shown for all the selected four drug candidates betamethasone phosphate, fluticasone, dexamethasone, remedesivir in Figures 3,4,5,6 respectively. The number and types of bonds formed in different modes of bonding interaction in 2D pictures are summarized in Fig.7.

Comparison of protein-ligand binding interactions with graphs: The protein-ligand binding interactions are studied for selected comparable drug candidates like betamethasone phosphate, fluticasone, dexamethasone with remedesivir. It is also revealed the interaction of specific amino acids that take part in the drug-protein interactions. All the docked structures were visualized by Discovery Studio Visualizer 2020. The visualized 3D pictures of sitting of ligand in protein structure and 2D pictures of bonding interactions are shown for all the selected four drug candidates betamethasone phosphate, fluticasone, dexamethasone, remedesivir in Figures 3,4,5,6 respectively. The number and types of bonds formed in different modes of bonding interaction in 2D pictures are summarized in Fig.7.

From the binding affinity Table1 \& Fig.1 and bonding interaction Fig.7, it is clearly understood that betamethasone phosphate has shown more binding affinity than remedesivir and comparatively more stable in all the ten modes as per the binding affinity values. Even though remedesivir has shown a greater number of pi-alkyl, alkyl binding interactions with protein binding sites, the presence of strong F-hydrogen bonding and F-pi bond interactions are predominant in case of betamethasone phosphate, a corticosteroid. The presence of alpha, beta unsaturated keto group in betamethasone phosphate is also responsible for its more binding affinity when compared to remedesivir. 
FIGURE: 3. Binding interactions of Betamethasone Phosphate with Protein: All the $\mathbf{1 0}$ modes of binding interactions are taken for discussion

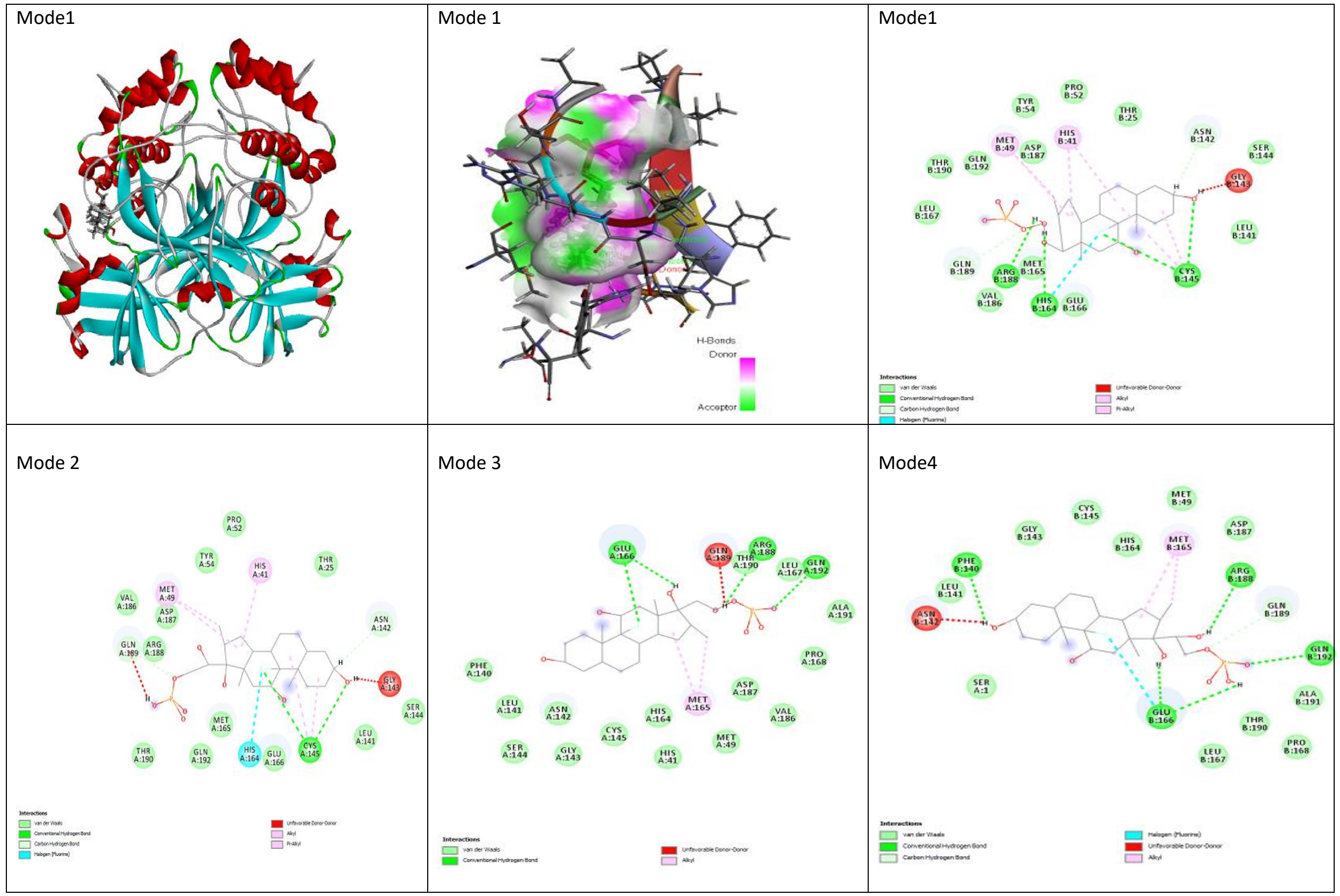




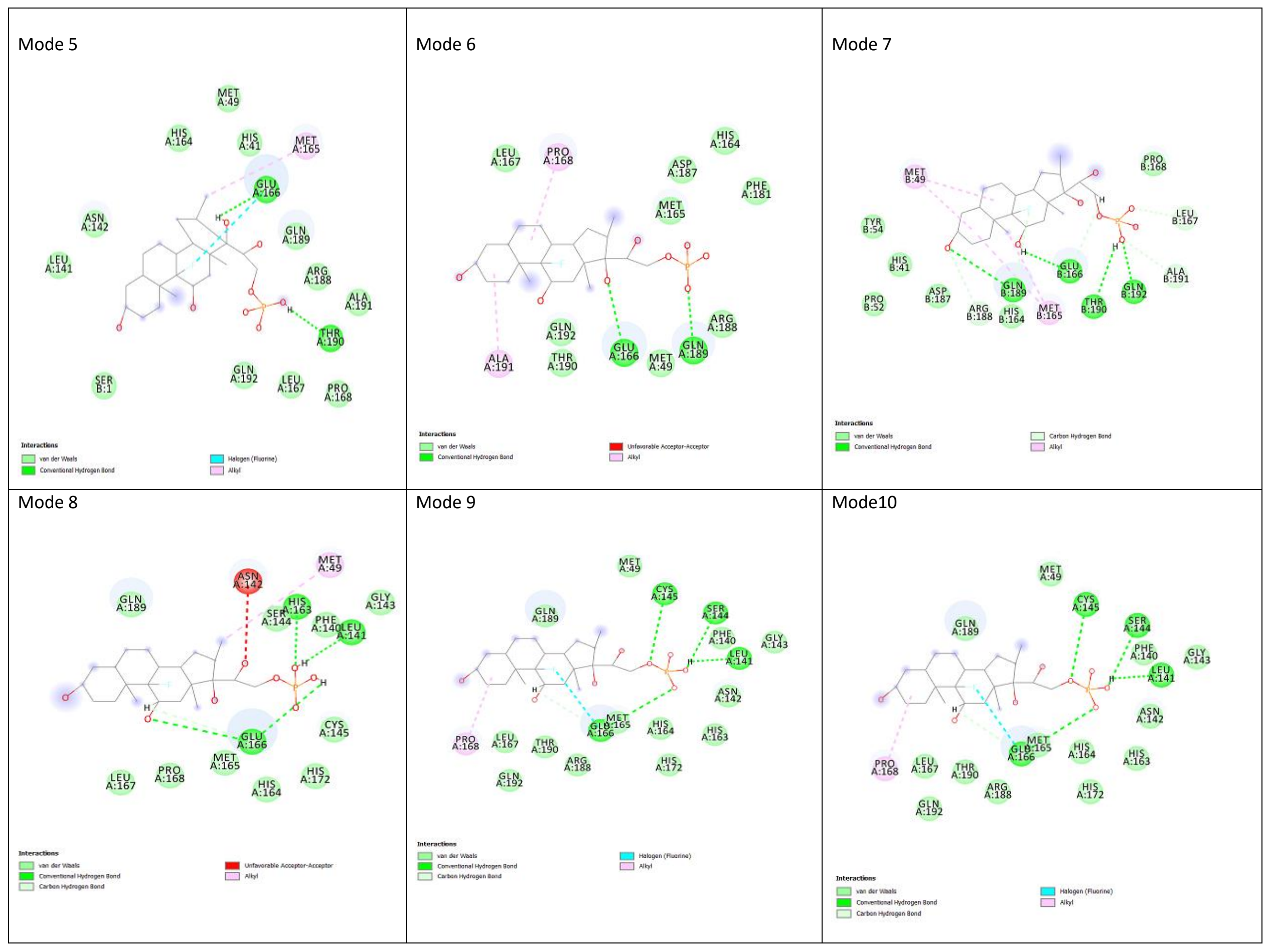


FIGURE: 4. Binding interactions of Fluticasone with Protein: All the 10 modes of binding interactions are taken for detailed discussion.

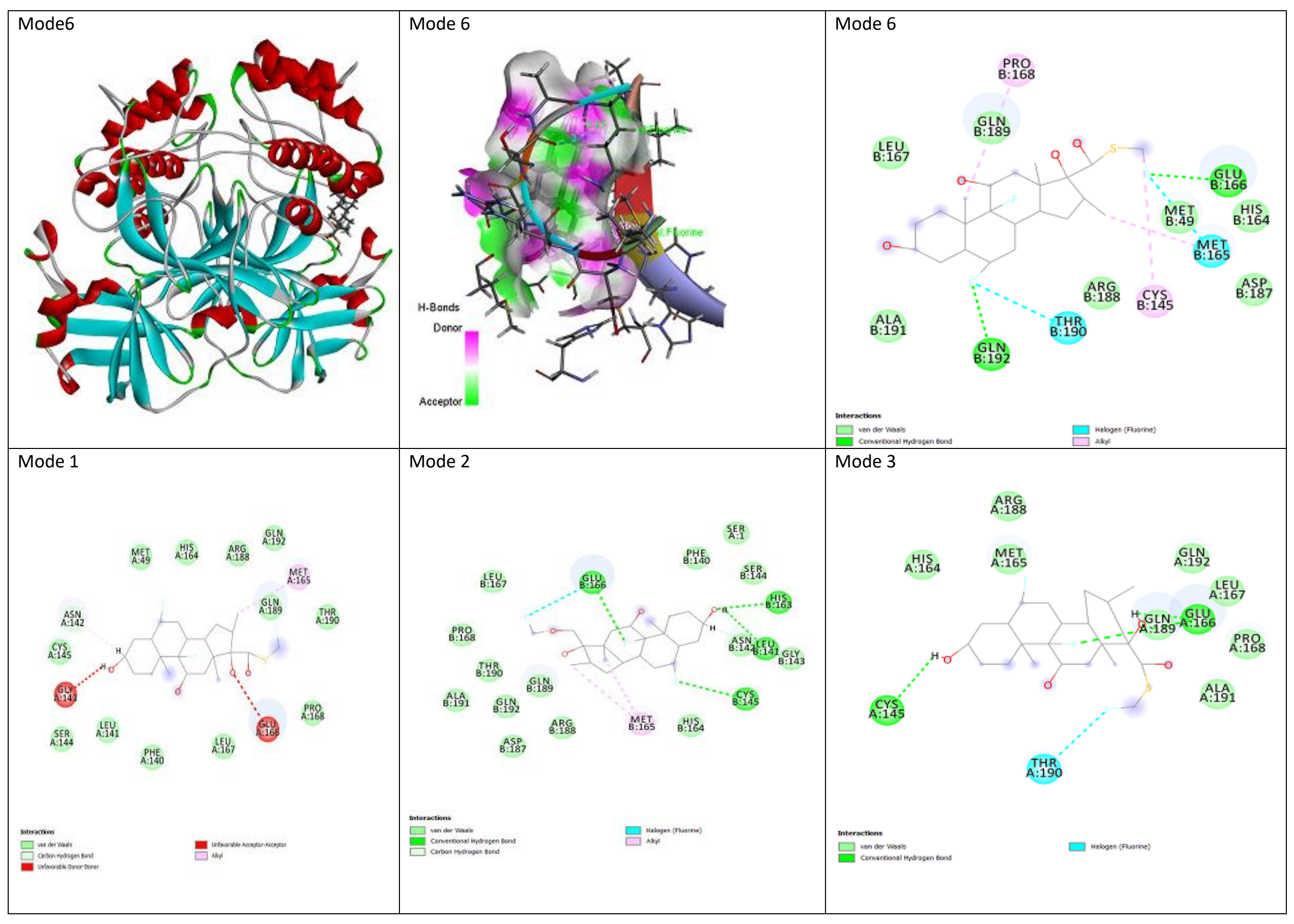




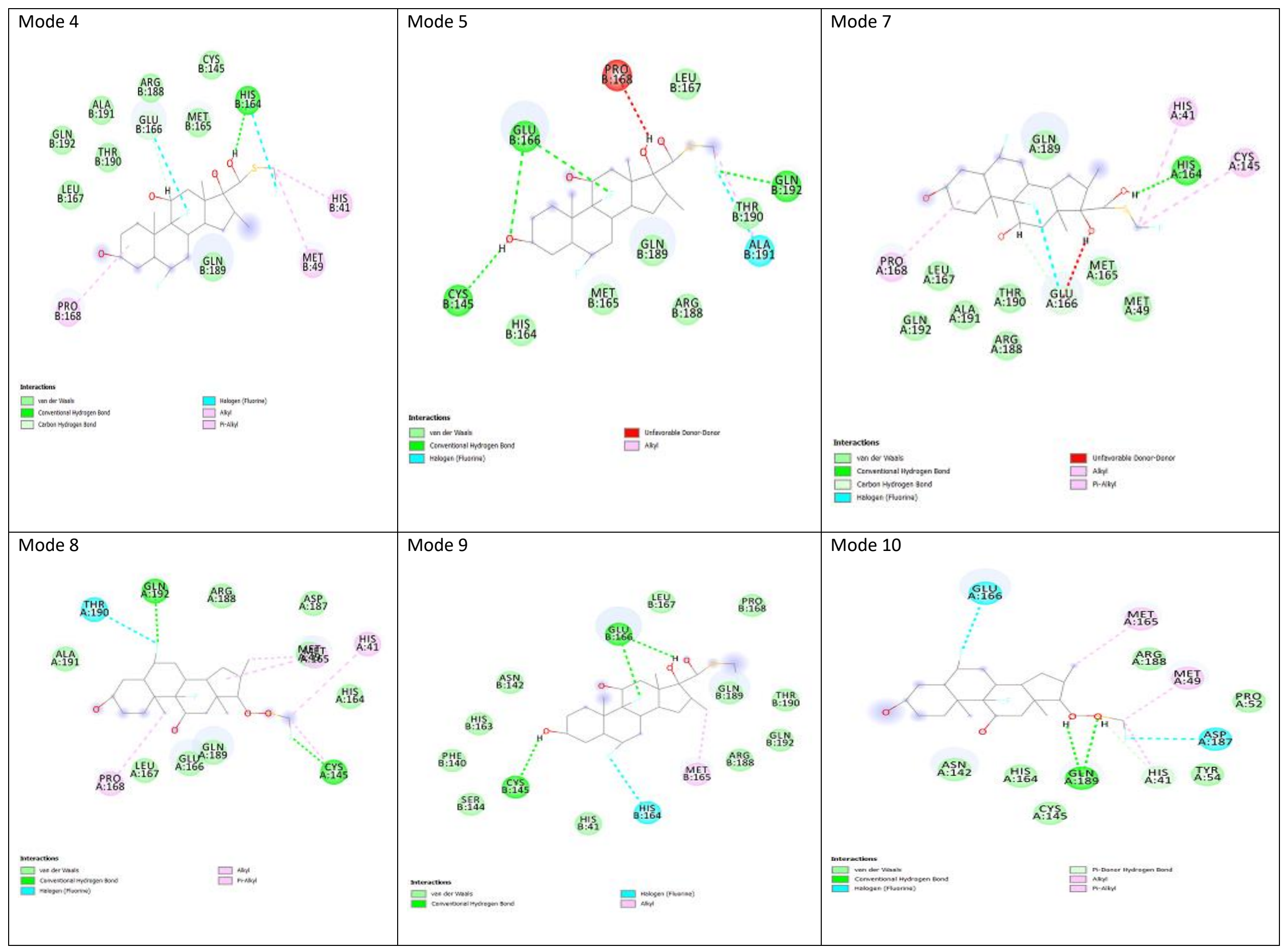


FIGURE: 5. Binding interactions of Dexamethasone with Protein: All the $\mathbf{1 0}$ modes of binding interactions are taken for detailed discussion.

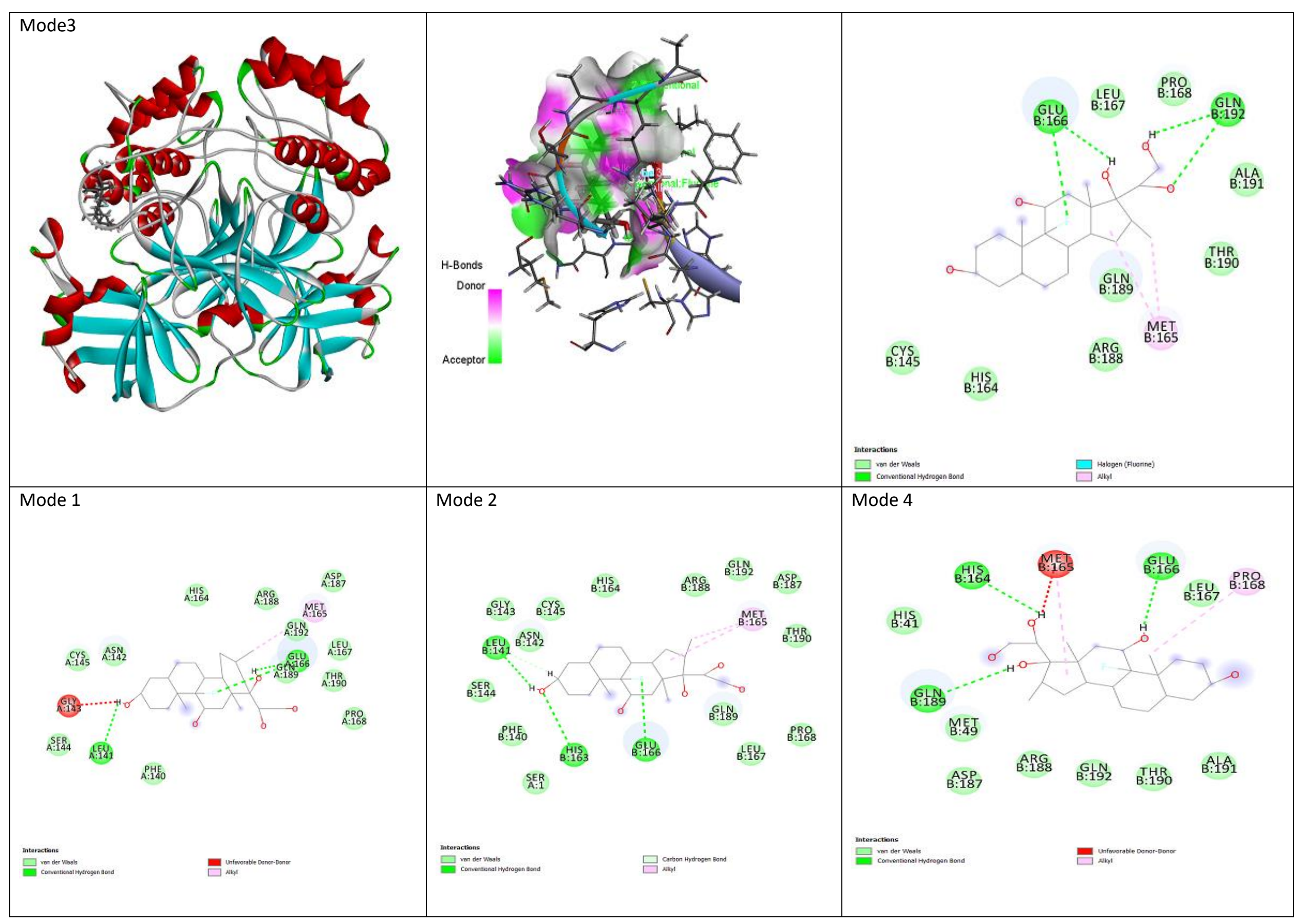




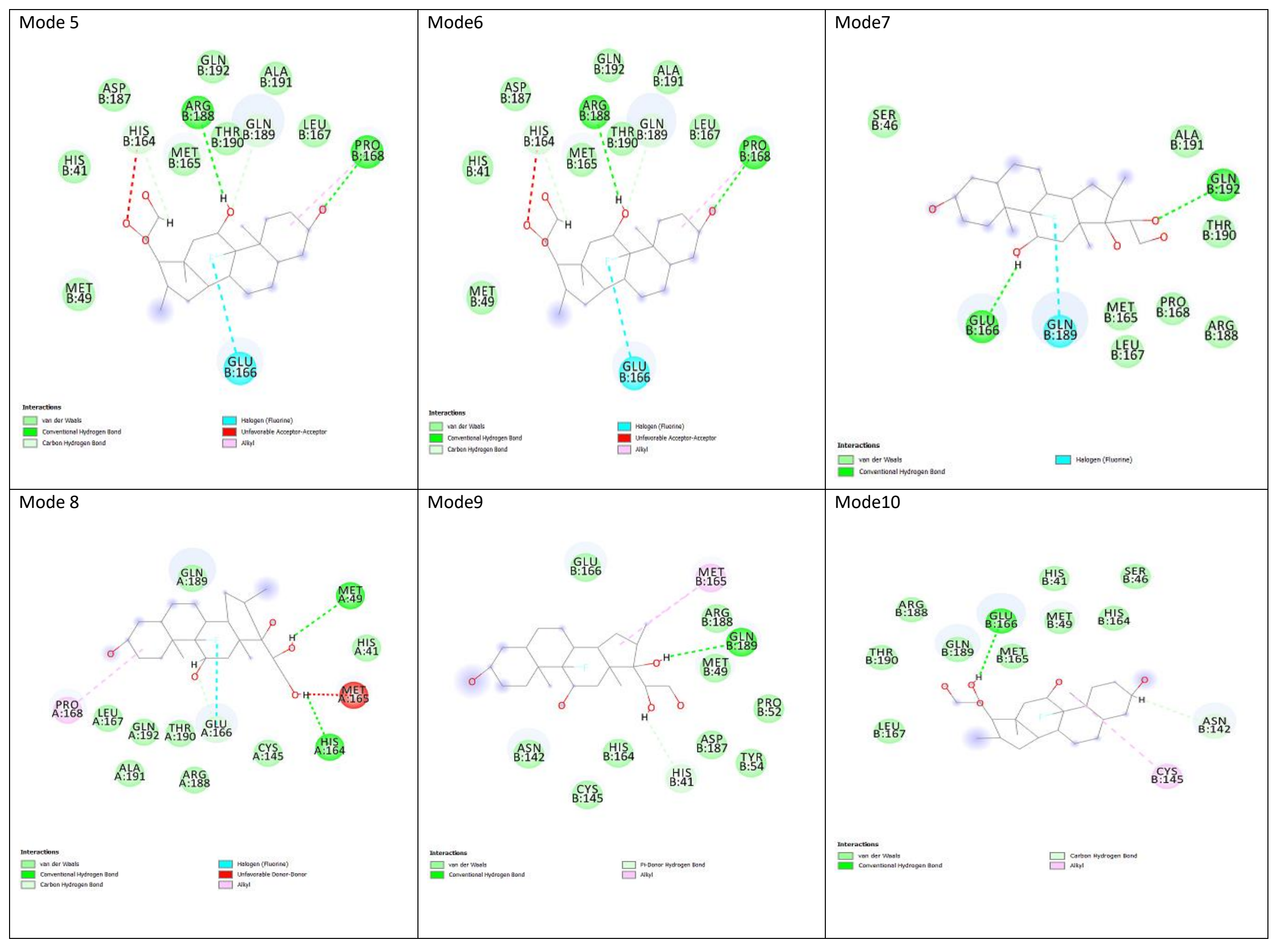


FIGURE: 6. Binding interactions of Remedesivir with Protein: All the $\mathbf{1 0}$ modes of binding interactions are taken for detailed discussion.

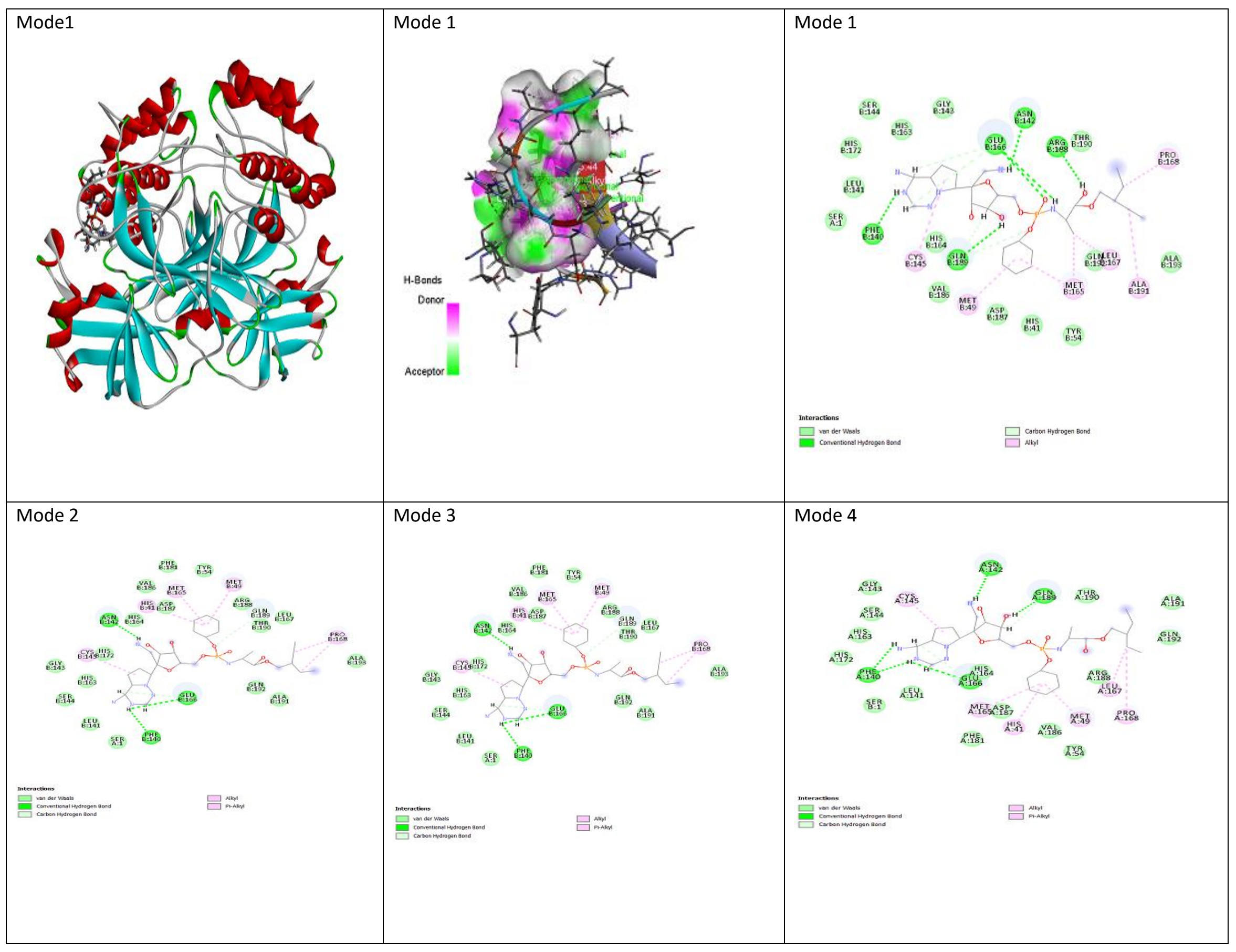




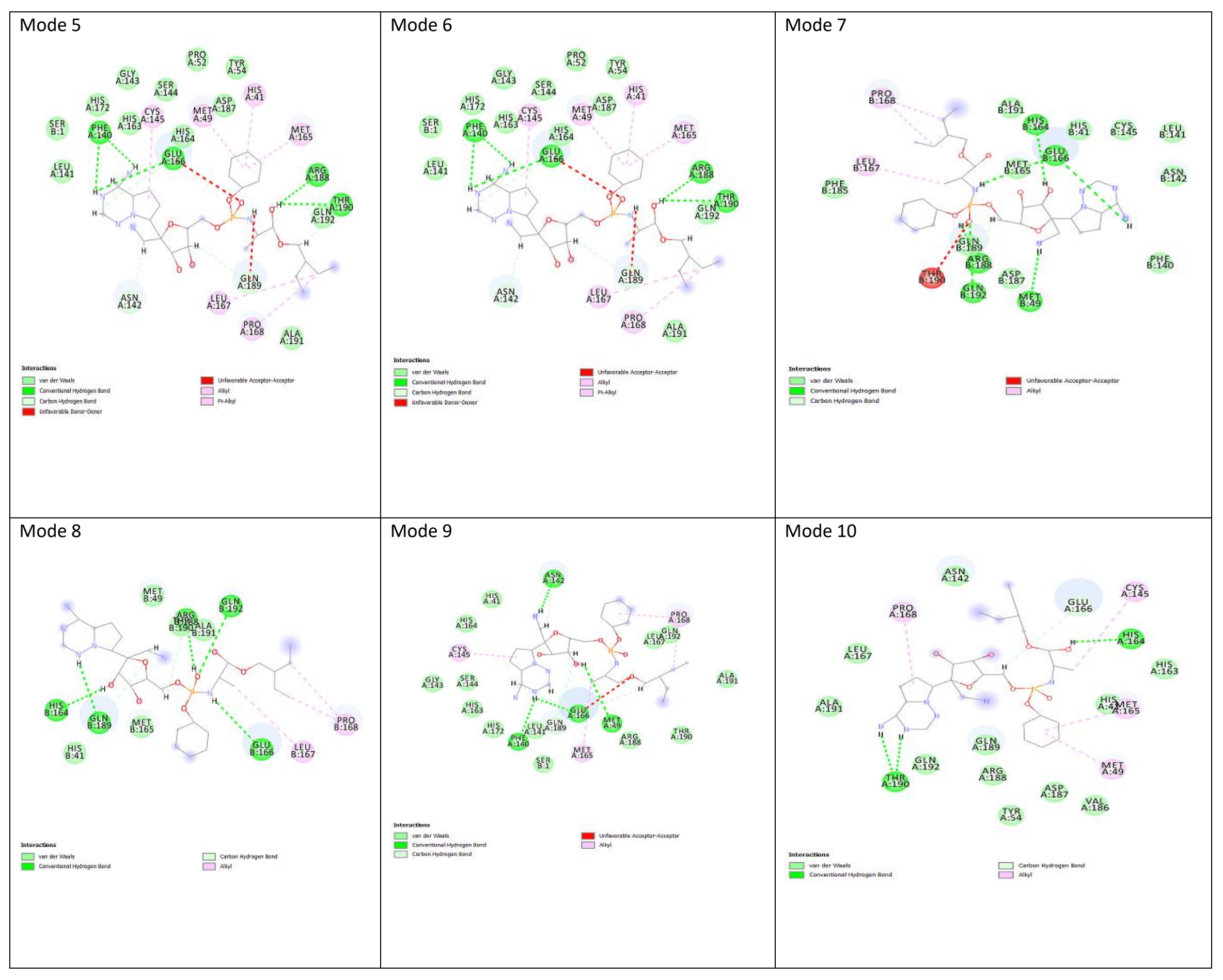


PROTIEN-LIGAND INTERACTIONSH+F :\# of Hydrogen and Fluorine bonds, F- \# of Fluorine bonds, Pi/Alkyl: \# of Pi/Alkyl

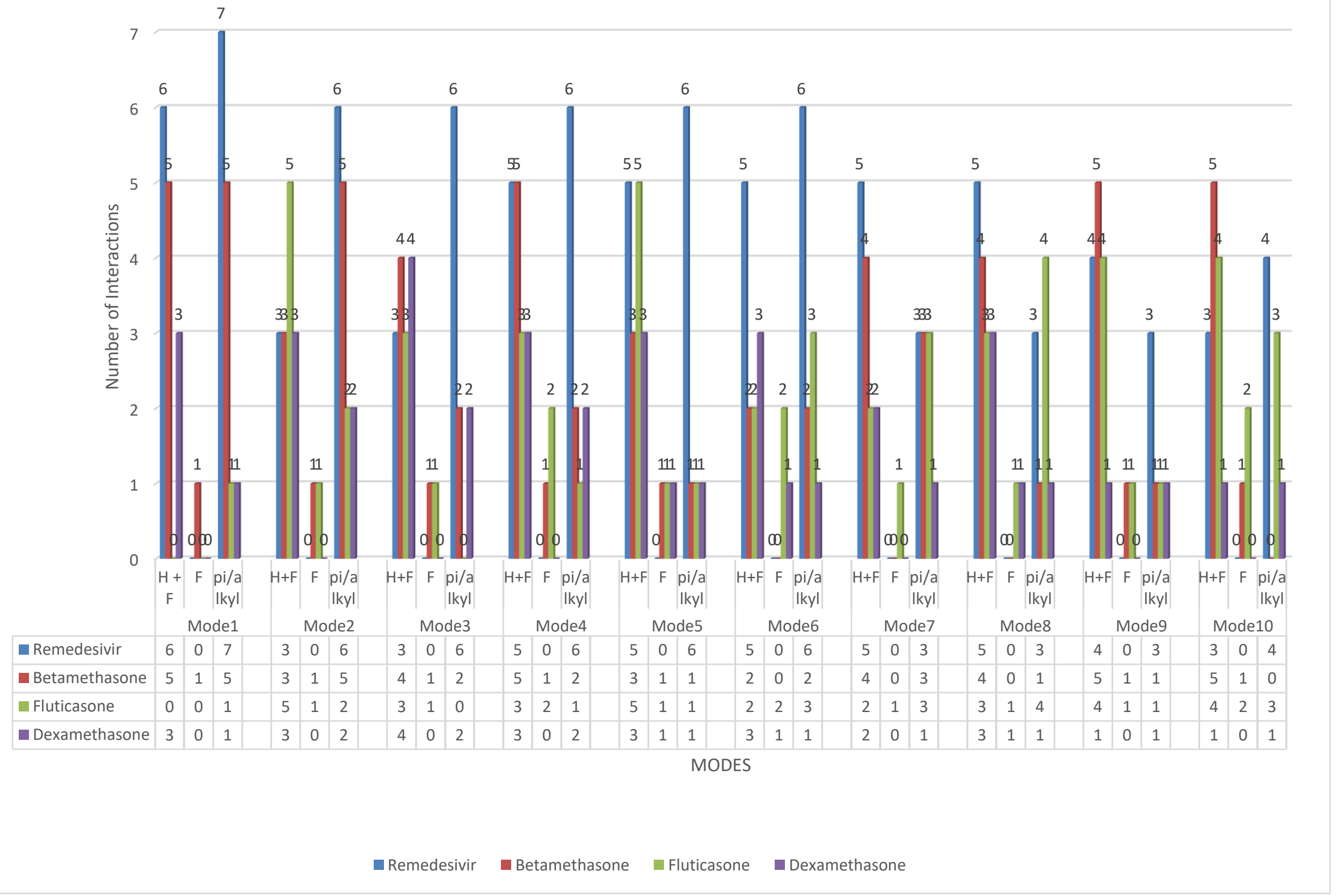

Fig. 7. Protein-Ligand interactions in 10 modes and number of different types of bonds formed. 
The presence of F-hydrogen bonding and F-pi interactions and also the presence of alpha, beta unsaturated ketone in case of other two corticosteroid ligands like Fluticasone and Dexamethasone are responsible for showing binding affinity closer to remedesvir, lopinavir and also more binding affinity than other drug candidates docked in the study.

Fluticasone is an inhaler drug used in patients with obstructive pulmonary diseases (e.g. asthma or COPD). Fluticasone propionate is an optimized drug for inhalation. Inhaled drugs are the mainstay of treatment in the care of pulmonary diseases such as asthma and COPD [20-22]. Compared with other routes of administration, respiratory drugs that are specifically designed for inhalation can offer. significant benefits, including direct delivery to the disease target site, rapid onset of action, high and long-term pulmonary efficacy, and reduced risk of systemic side effects [23]. It can be readily used by the people moving in more infected areas where the COVID-19 infection is severely spread. It can also be used as preventive treatment upon the doctors advise and it is easy to carry inhaler. It needs further clinical studies to use this as inhaler or intravenous administration for preventive and cure measures of COVID-19.

Conclusions: Based on the above results, it is clear that three drug candidates betamethasone phosphate, fluticasone and dexamethasone approved by FDA for other medical purposes, have high potential bond affinity and binding interactions against SARS-CoV-2 protease on par with the drugs currently using for COVID-19 treatment. It needs further clinical studies to suggest them as alternative medical treatments for COVID-19. 


\section{References}

1. Mcconkey B, Sobolev V, Edelman M. The performance of current methods in ligand-protein docking. Curr Sci;83.

2. Jorgensen WL. The many roles of computation in drug discovery. Science 2004;303:1813-8.

3. Bajorath J. Integration of virtual and high-throughput screening. Nat Rev Drug Discov 2002;1:882-94.

4. Langer T, Hoffmann RD. Virtual screening: an effective tool for lead structure discovery? Curr Pharm Des 2001;7:509-527.

5. Kitchen DB, Decornez H, Furr JR, Bajorath J. Docking and scoring in virtual screening for drug discovery: methods and applications. Nat Rev Drug Discov 2004;3:935-949.

6. Li G, De Clercq E. Therapeutic options for the 2019 novel coronavirus (2019-nCoV). Nature reviews. Drug discovery 2020;19:149-50.

7. Lim J, Jeon S, Shin HY, et al. Case of the Index Patient Who Caused Tertiary Transmission of COVID-19 Infection in Korea: the Application of Lopinavir/Ritonavir for the Treatment of COVID-19 Infected Pneumonia Monitored by Quantitative RT

PCR. J Korean Med Sci 2020;35:e79.

8. Holshue ML, DeBolt C, Lindquist S, et al. First Case of 2019 Novel Coronavirus in the United States. N Engl J Med 2020;382:929-36.

9. Wang $M$, Cao R, Zhang $L$, et al. Remdesivir and chloroquine effectively inhibit the recently emerged novel coronavirus (2019-nCoV) in vitro. Cell Res 2020;30:269-71.

10. Yao X, Ye F, Zhang M, et al. In Vitro Antiviral Activity and Projection of Optimized Dosing Design of Hydroxychloroquine for the Treatment of Severe Acute Respiratory Syndrome Coronavirus 2 (SARS-CoV-2). Clin Infect Dis an Off Publ Infect Dis Soc Am [Epub ahead of print].

11. Lv Z, Chu Y, Wang Y. HIV protease inhibitors: a review of molecular selectivity and toxicity. HIV AIDS (Auckl) 2015;795-104.

12. Lu R, Zhao X, Li J, et al. Genomic characterisation and epidemiology of 2019 novel coronavirus: implications for virus origins and receptor binding. Lancet (London, England) 2020;395:565-74.

13. Wan Y, Shang J, Graham R, Baric RS, Li F. Receptor Recognition by the Novel Coronavirus from Wuhan: an Analysis Based on Decade-Long Structural Studies of SARS Coronavirus. J Virol;94.

14. Three drugs fairly effective on novel coronavirus at cellular level - Xinhua | English.news.cn http://www.xinhuanet.com/ english/2020-01/30/c_138742163.htm Accessed: 2020-04-11.

15. Coronavirus outbreak: Cocktail of flu, HIV drugs appears to help fight virus, say Thai doctors World News https://www. indiatoday.in/world/story/coronavirus-outbreak-cocktail-fluhiv-drugs-treatment-thai-doctors1642783-2020-02-03 Accessed: 2020-04-11. 
16. Singh AK, Singh A, Shaikh A, Singh R, Misra A. Chloroquine and hydroxychloroquine in the treatment of COVID-19 with or without diabetes: A systematic search and a narrative review with a special reference to India and other developing countries. Diabetes Metab Syndr [Epub ahead of print].

17. Warui DM, Baranger AM. Identification of specific small molecule ligands for stem loop 3 ribonucleic acid of the packaging signal Psi of human immunodeficiency virus-1. J Med Chem 2009;52:5462-473.

18. Cosconati S, Marinelli L, Trotta R, et al. Tandem application of virtual screening and NMR experiments in the discovery of brand new DNA quadruplex groove binders. J Am Chem Soc 2009;131:16336-7.

19. Trott O, Olson AJ. AutoDock Vina: improving the speed and accuracy of docking with a new scoring function, efficient optimization, and multithreading. J Comput Chem 2010;31:455-61.

20. F. Lavorini, C. Mannini, and E. Chellini, "Challenges of inhaler use in the treatment of asthma and chronic obstructive pulmonary disease," EMJ Respiratory, vol. 3, pp. 98-105, 2015.

21. Global Initiative for Asthma, GINA Report: Global Strategy for Asthma Management and Prevention, 2017, http://ginasthma.org/2017-gina-report-global-strategy-forsthmamanagement-and-prevention/.

22. Global Initiative for Chronic Obstructive Lung Disease, Global Strategy for the Diagnosis, Management, and Prevention of Chronic Obstructive Pulmonary Disease, 2017, http://goldcopd.org/gold-2017-globalstrategy-diagnosismanagement-prevention-copd/].

23. J. Wright, D. Brocklebank, and F. Ram, "Inhaler devices for the treatment of asthma and chronic obstructive airways disease (COPD)," Quality and Safety in Health Care, vol. 11,no. 4, pp. 376-382, 2002.

24. Wishart DS, Feunang YD, Guo AC, Lo EJ, Marcu A, Grant JR, Sajed T, Johnson D, Li C, Sayeeda Z, Assempour N, lynkkaran I, Liu Y, Maciejewski A, Gale N, Wilson A, Chin L, Cummings R, Le D, Pon A, Knox C, Wilson M. DrugBank 5.0: a major update to the DrugBank database for 2018. Nucleic Acids Res. 2017 Nov 8. doi: 10.1093/nar/gkx1037. 